\title{
STABILITY IMPLICATIONS ON THE ASYMPTOTIC BEHAVIOR OF NONLINEAR SYSTEMS
}

\section{KUO-LIANG CHIOU}

Department of Mathematics

Wayne State University

Detroit, Michigan 48202

(Received July 20, 1978)

ABSTRACT. In this paper we generalize Bownds' Theorems (1) to the systems $\frac{d Y(t)}{d t}=$ $A(t) Y(t)$ and $\frac{d X(t)}{d t}=A(t) X(t)+F(t, X(t))$. Moreover, we also show that there always exists a solution $X(t)$ of $\frac{d X}{d t}=A(t) X+B(t)$ for which $\underset{t \rightarrow \infty}{\lim \sup }|| X(t) \|>$ $O(=\infty)$ if there exists a solution $Y(t)$ for which $\underset{t \rightarrow \infty}{\lim \sup }|| Y(t) \|>0(=\infty)$.

KEY WOKDS AND PHRASES. stable, norm, linear systems, null solution, SchauderTychorofb Theorem, uniformly converges, equicontenuous.

1980 MATHEMATICS SUBJECT CLASSIFICATION CODES. Primary 34005, 34010, 34C10, Secondary 34A30, 34CTS, 34020 .

1. INTRODUCTION.

In this paper we shall study the stability behavior of the following systems

$$
\frac{d Y(t)}{d t}=A(t) Y(t), 0 \leq t<\infty
$$

and

$$
\frac{d X(t)}{d t}=A(t) X(t)+F(t, X(t)), \quad 0 \leq t<\infty
$$

where $A(t)$ is a continuous matrix on $R^{n}$ for all $0 \leq t<\infty, F(t, X(t))$ is a real valued continuous $n$-vector defined on $[0, \infty) X R^{n}$ and $X(t)$ and $Y(t)$ are $n$-vectors.

Consider special equations of (1.1) and (1.2)

$$
y^{\prime \prime}+a(t) y=0, \quad 0 \leq t<\infty
$$

and

$$
x^{\prime \prime}+a(t) x=g\left(t, x, x^{\prime}\right), \quad 0 \leq t<\infty
$$


where $a(t) \in C[0, \infty)$ and $g\left(t, x, x^{\prime}\right)$ is continuous on $[0, \infty) \times R \times R$. From some theorems of stability theory, Bownds [1] showed that (1.3) has a solution $y(t)$ with property

$$
\underset{t \rightarrow \infty}{\lim \sup _{t \rightarrow \infty}}\left(|y(t)|+\left|y^{\prime}(t)\right|\right)>0
$$

He also established that (1.4) has the property (1.5) provided that the zero solution of (1.3) is stable and there exists a function $\gamma(t) \in L[0, \infty)$ such that

$$
\left|g\left(t, x, x^{\prime}\right)\right| \leq \gamma(t) \quad\left(|x|+\left|x^{\prime}\right|\right)
$$

for $\left(t, x, x^{\prime}\right) \in[0, \infty) \times R \times R$.

Thus in the following section we shall extend the above results to systems (1.1) and (1.2). In section 3 we shall consider a nonhomogeneous system

$$
\frac{d X(t)}{d t}=A(t) X(t)+B(t), \quad 0 \leq t<\infty
$$

where $B(t)$ is a continuous vector for $0 \leq t<\infty$. We shall prove that there always exists a solution $X(t)$ of (1.6) for which $\lim _{t \rightarrow \infty}|| X(t) \|>0(=\infty)$, if there exists a solution $Y(t)$ of $(1.1)$ for which $\underset{t \rightarrow \infty}{\lim \sup _{t \rightarrow \infty}}\|\mathrm{Y}(\mathrm{t})\|>0(=\infty)$. Here $\|\cdot\|$ is an appropriate vector (or matrix) norm.

2. ASYMPTOTIC BEHAVIOR FOR (1.1) AND (1.2).

Before stating main theorems, let us recall a theorem from Coppel [2, p. 60]. THEOREM 2.1. (Hartman [2, p. 60]). Suppose that, for every solution $Y(t)$ of (1.1), the limit

$$
\lim _{t \rightarrow \infty}|| Y(t)||
$$

exists and is finite. If there exists a nontrivial solution $Y(t)$ of (i.1) for which the limit (2.1) is zero, then

$$
\int_{t_{0}}^{t} t_{r} A(s) d s \rightarrow-\infty \quad \text { as } t \rightarrow \infty .
$$

From the above theorem we will obtain the following corollary which is a generalization of Theorem 1 in [1].

COROLLARY 2.1. Suppose that

$$
\int_{t_{0}}^{\infty} t_{r} A(s) d s<\infty .
$$

Then there exists a nontrivial solution $Y(t)$ of (1.1) for which 


$$
\lim _{t \rightarrow \infty} \sup |y(t)| \mid>0
$$

PROOF. Suppose, to the contrary, that all solutions $Y(t)$ of (1.1) satisfy

$$
\lim _{t \rightarrow \infty}|| Y(t)||=0
$$

From Theorem 2.1 we obtain $\int_{t_{0}}^{t} t_{r} A(s) d s \rightarrow-\infty$ as $t \rightarrow \infty$. This leads to a contradiction. The corollary then follows.

Throughout this paper we shall denote $\Phi(t)$, the fundamental matrix of (1.1) with initial condition $\Phi(0)=$ I (identity matrix).

Now we shall prove the following theorem via the Schauder-Tychonoff Theorem [2, p. 9]

THEOREM 2.2. Suppose that the null solution of (1.1) is stable and that there exists a solution $Y(t)$ of $(1.1)$ for which

$$
\lim _{t \rightarrow \infty}|| Y(t)||>0
$$

Suppose also that there exists $\gamma(t) \in L_{1}\left[t_{0}, \infty\right)$ such that for some positive constant $\ell$

$$
|| F(t, x)|| \leq \gamma(t)|| x||^{\ell}
$$

Then there exists a nontrivial solution $X(t)$ of (1.2) for which

$$
\lim _{t \rightarrow \infty} \sup || x(t) \|>0
$$

PROOF. Since the null solution of (1.1) is stable, there exists a positive constant $\mathrm{k}$ such that

$$
|| \Phi(t) \Phi^{-1}(s)|| \leq k
$$

for all $0 \leq t \leq s$ and there exists a nontrivial solution $Y(t)$ of (1.1) for which (2.2) holds and

$$
\|\mathrm{Y}(\mathrm{t})\| \leq 1-\varepsilon
$$

for $t \geq t_{0}$ and for given small positive constant $\varepsilon(<1)$.

Since $\gamma(t) \in L_{1}\left[t_{0}, \infty\right)$, there exists $T_{0}\left(>t_{0}\right)$ such that

$$
k \int_{t}^{\infty} \gamma(s) d s<\varepsilon \quad \text { for all } t \geq T_{o} \text {. }
$$

Via the Schauder-Tychonoff Theorem we shall establish the existance of a solution 
of the integral equation

$$
X(t)=Y(t)-\Phi(t) \quad \int_{t}^{\infty} \Phi^{-1}(s) f(s, X(s)) d s, t \geq T_{0} .
$$

Consider the set

$$
\begin{gathered}
F=\left\{U ; U(t)=X(t) \text { is continuous on } J_{0}=\left[T_{0}, \infty\right)\right. \text { and } \\
\left.|| U(t) \| \leq 1 \text { for } t \geq T_{0}\right\}
\end{gathered}
$$

and define the operator $\mathrm{T}$ by

$$
\mathrm{TU}(\mathrm{t})=\mathrm{Y}(\mathrm{t})-\int_{t}^{\infty} \Phi(t) \Phi^{-1}(\mathrm{~s}) \mathrm{f}(\mathrm{s}, \mathrm{U}(\mathrm{s})) \mathrm{ds} .
$$

First, we shall show that $\mathrm{TF} \subset \mathrm{F}$. Taking the norm to both sides of (2.8) and using (2.3), (2.4), (2.5), and (2.6), we obtain for $U \in F$

$$
\begin{aligned}
\|\mathrm{TU}(\mathrm{t})\| & \leq\|\mathrm{Y}(\mathrm{t})\|+\int_{\mathrm{t}}^{\infty}\left\|\Phi(\mathrm{t}) \Phi^{-1}(\mathrm{~s}) \mathrm{f}(\mathrm{s}, \mathrm{U}(\mathrm{s}))\right\| \mathrm{ds} \\
& \leq 1-\varepsilon+\mathrm{k} \int_{\mathrm{t}}^{\infty}\|\mathrm{f}(\mathrm{s}, \mathrm{U}(\mathrm{s}))\| \mathrm{ds} \\
& \leq 1-\varepsilon+\mathrm{k} \int_{\mathrm{t}}^{\infty} \gamma(\mathrm{s})\|\mathrm{U}(\mathrm{s})\|^{\ell} \mathrm{ds} \\
& \leq 1-\varepsilon+\mathrm{k} \int_{\mathrm{t}}^{\infty} \gamma(\mathrm{s}) \mathrm{ds} \\
& \leq 1-\varepsilon+\varepsilon=1 .
\end{aligned}
$$

It is clear that $\mathrm{TU}(t)$ is continuous on $\mathrm{J}_{0^{\circ}}$. This proves $\mathrm{TF} \subset \mathrm{F}$.

Second, we shall show that $t$ is continuous. Suppose that the sequence $\left\{U_{n}\right\}$ in $F$ converges uniformly to $U$ in $F$ on every compact subinterval of $J_{0}$. We claim that $\mathrm{TU}_{\mathrm{n}}$ converges uniformly to $\mathrm{TU}$ on every compact subinterval of $\mathrm{J}_{0} \cdot$ Let $\epsilon_{1}$ be a small positive number satisfying $\epsilon_{1}<1$. Since $\gamma(t) L_{1}\left[t_{0}, \infty\right)$, there exists $\mathrm{T}_{1}>\mathrm{T}_{0}$ so that for $\mathrm{t} \geq \mathrm{T}_{1}$

$$
k \int_{t}^{\infty} \gamma(s) d s<\frac{\epsilon}{4} .
$$

By the uniform convergence, there is an $N=N\left(\epsilon_{1}, T_{1}\right)$ such that if $n \geq N$, then

$$
|| \mathrm{f}\left(\mathrm{s}, \mathrm{U}_{\mathrm{n}}(\mathrm{s})\right)-\mathrm{f}(\mathrm{s}, \mathrm{U}(\mathrm{s}))||<\frac{\epsilon_{1}}{2 \mathrm{kT}_{1}}, \mathrm{~T}_{\mathrm{o}} \leq \mathrm{s} \leq \mathrm{T}_{1} \text {. }
$$

Then using $(2.8),(2.9),(2.10),(2.3),(2.4)$, and the fact that ||$U_{n}(t)|| \leq 1$ and $\|\mathrm{U}(\mathrm{t})\| \leq 1$ for $\mathrm{T}_{\mathrm{O}} \leq t<\infty$, we obtain the following inequalities 


$$
\begin{aligned}
& \left\|T U_{n}(t)-\mathrm{IU}(t)\right\|=\| \int_{t}^{\infty} \Phi(t) \Phi^{-i}(s) f\left(s, U_{n}(s)\right) d s-\int \Phi(t) \Phi^{-1}(s) f(s, U(s) d s \| \\
& \leq \int_{t}^{\mathrm{T}}\left\|\Phi(t) \Phi^{-1}(s)\right\|\left\|f\left(s, U_{n}(s)\right)-f(s, U(s))\right\| d s \\
& +\int_{\mathrm{T}_{1}}^{\infty}\left\|\Phi(\mathrm{t})_{\Phi}^{-1}(\mathrm{~s})\right\|\left\|\mathrm{f}\left(\mathrm{s}, \mathrm{U}_{\mathrm{n}}(\mathrm{s})\right)\right\| \mathrm{ds}+\int_{\mathrm{T}_{1}}^{\infty}\left\|\Phi(\mathrm{t}) \Phi^{-1}(\mathrm{~s})\right\|\|\mathrm{f}(\mathrm{s}, \mathrm{U}(\mathrm{s}))\| \mathrm{ds} \\
& \leq k \int_{t}^{T_{1}}|| f\left(s, U_{n}(s)\right)-f(s, U(s)) \| d s+2 k \int_{T_{1}}^{\infty} \gamma(s) d s \\
& <\frac{\epsilon_{1}}{2}+\frac{\varepsilon_{1}}{2}=\epsilon_{1} \quad \text { for } \mathrm{n} \geq \mathrm{N}
\end{aligned}
$$

This shows that $\mathrm{TU}_{\mathrm{n}}$ converges uniformly to $\mathrm{TU}$ on every compact subinterval of $\mathrm{J}_{\mathrm{O}}$. Hence $\mathrm{T}$ is continuous.

Third, we claim that the functions in the image set $\mathrm{TF}$ are equicontinuous and bounded at every point of $\mathrm{J}_{0^{\circ}}$. Since $\mathrm{TF} \subset \mathrm{F}$, it is clear that the functions in $\mathrm{TF}$ are uniformly bonded. Now we show that they are equicontinuous at eact point of $J_{0}$. For each $U \in F$, the function $z(t)=T U(t)$ is a solution of the linear system

$$
\frac{d V}{d t}=A(t) V+f(t, U(t))
$$

Since $\|z(t)\|=|| T U(t) \| \leq 1$ and $\|f(t, U(t))\|$ is uniformly bounded for $U \in F$ on any finite $t$ interval, we see that $\frac{d z}{d t}$ is uniformly bounded on any finite interval. Therefore, the set of all such $\mathrm{z}$ is equicontinuous at each point of $\mathrm{J}_{\mathrm{o}}$ (see [2, p.6]).

A11 of the hypotheses of the Schauder-Tychonoff Theorem are satisfied. Thus there exists a $U \in F$ such that $U(t)=T U(t)$; that is, there exists a solution $X(t)$ of

$$
X(t)=Y(t)-\Phi(t) \int_{t}^{\infty} \Phi^{-1}(s) f(s, x(s)) d s
$$

Thus, from the hypotheses and the above equality, we obtain.

$$
\lim _{t \rightarrow \infty} \sup |X(t)-Y(t)| \mid=0
$$

Since $\underset{t \rightarrow \infty}{\lim \sup _{t \rightarrow \infty}}|| Y(t) \|>0,(2.11)$ implies that $\underset{t \rightarrow \infty}{\lim \sup }|| X(t) \|>0$. This proves the theorem.

It is clear that $(1.4)$ can be written as the form (1.2) with 


$$
A(t)=\left(\begin{array}{ll}
0 & 1 \\
-a(t) & 0
\end{array}\right) \text { and } F(t, X)=\left(\begin{array}{l}
0 \\
g\left(t, x, x^{\prime}\right)
\end{array}\right)
$$

where $x=\operatorname{colum}\left(x, x^{\prime}\right)$. Thus we can apply Theorem 2.2 to (1.4) to obtain the following corollary which is a generalization of Theorem 2 in [1].

COROLLARY 2.2. Suppose that the null solution of (1.3) is stable and that there exists $Y(t) \in L_{1}\left[t_{0}, \infty\right)$ such that for some positive constant $\ell$

$$
\| \mathrm{g}\left(\mathrm{t}, \mathrm{x}, \mathrm{x}^{\prime}\right)|| \leq \gamma(\mathrm{t})\left(|\mathrm{x}|+\left|\mathrm{x}^{\prime}\right|\right)^{\ell} \text {. }
$$

Then there exists a nontrivial solution $x(t)$ of $(1.4)$ for which

$$
\lim _{t \rightarrow \infty}\left(|x|+\left|x^{\prime}\right|\right)>0 \text {. }
$$

PROOF. Since ${ }_{r} \mathrm{~A}(\mathrm{t})=0$ for Corollary 2.1, we know that there exists a so1ution $Y(t)$ of (1.1) for which

$$
\lim _{t \rightarrow \infty}|| Y(t)||>0 .
$$

If we take ||$x||=|x|+\left|x^{\prime}\right|$, then the corollary follows from Theorem 2.2.

\section{ASYMPTOTIC BEHAVIOR FOR (1.6).}

In this section we shall show that if there exists a solution $Y(t)$ of (1.1) for which $\underset{t \rightarrow \infty}{\lim \sup }\|Y(t)\|>0(=\infty)$, then there exists a solution $X(t)$ of $(1.6)$ for which $\lim _{t \rightarrow \infty}|| x(t)||>0(=\infty)$.

THEOREM 3.1. Suppose that there exists a solution $Y(t)$ of (1.1) for which

$$
\limsup _{t \rightarrow \infty}|| Y(t)||>0 \text {. }
$$

Then there exists a solution $X(t)$ of (1.6) for which

$$
\lim _{t \rightarrow \infty} \sup _{1} \mid \mathrm{X}(\mathrm{t}) ! !>0 \text {. }
$$

PROOF. From the variation of constants formula we know that any solution $X(t)$ of (1.6) can be written as the form below

$$
\mathrm{X}(\mathrm{t})=\Phi(\mathrm{t}) \mathrm{c}+\Phi(\mathrm{t}) \int_{0}^{\mathrm{t}} \Phi^{-1}(\mathrm{~s}) \mathrm{B}(\mathrm{s}) \mathrm{ds}
$$

Hence we shall choose $c$ so that $Y(t)=\Phi(t) c$ satisfies (3.1).

First, let us suppose

$$
\underset{t \rightarrow \infty}{\lim \sup _{t \rightarrow \infty}}\left\|\Phi(t) \int_{0}^{t} \Phi^{-1}(s) B(s) d s\right\|>0 .
$$


Let $X_{1}(t)=X(t)-Y(t)$. It is clear that $X_{1}(t)$ is a solution of (1.6). Thus from (3.3) and (3.4) we obtain

$$
\begin{aligned}
\underset{t \rightarrow \infty}{\limsup } & \left\|X_{1}(t)\right\|=\underset{t \rightarrow \infty}{\lim \sup _{t \rightarrow \infty}}\|X(t)-Y(t)\| \\
& =\underset{t \rightarrow \infty}{\lim \sup }\left\|\Phi(t) \int_{0}^{t} \Psi^{-1}(s) B(s) d s\right\|>0 .
\end{aligned}
$$

Thus there exists a solution $X_{1}(t)$ of (1.6) for which (3.2) holds.

Second, suppose that

$$
\lim _{t \rightarrow \infty}\left\|\Phi(t) \int_{0}^{t} \Phi^{-1}(s) B(s) d s\right\|=0 .
$$

Taking the norm to both sides of (3.3) and using (3.1) and (3.5) we obtain

$$
\begin{aligned}
& \limsup _{t \rightarrow \infty}\|X(t)\| \geq \underset{t \rightarrow \infty}{\lim \sup _{t \rightarrow \infty}}\left(\|Y(t)\|-\left\|\Phi(t) \int_{0}^{t} \Phi^{-1}(s) B(s) d s\right\|\right) \\
& \underset{t \rightarrow \infty}{\limsup _{t \rightarrow \infty}}|| Y(t)\left\|-\underset{t \rightarrow \infty}{\lim \sup _{t \rightarrow \infty}}\right\| \Phi(t) \int_{0}^{t} \Phi^{-1}(s) B(s) d s \| \\
& \geq \lim _{t \rightarrow \infty}|| Y(t)||>0 .
\end{aligned}
$$

This shows that $X(t)$ satisfies (3.2). The theorem then follows.

Using the same argument as Theorem 3.1 we also can obtain the following theorem.

THEOREM 3.2. Suppose that there exists a solution $Y(t)$ of (1.1) for which

$$
\lim _{t \rightarrow \infty}\|Y(t)\|=\infty \text {. }
$$

Then there exists a solution $X(t)$ cf (1.6) for which

$$
\limsup _{t \rightarrow \infty}|| x(t) \|=\infty \text {. }
$$

PROOF. Since the proof is almost the same as Theorem 3.1, we shall omit the detail.

REMARKS. It is interesting to note that Hatvani and Pintér [3] have studied this type of problem for equation (1.4).

ACKNOWLEDGEMENTS. This work was supported by the U.S. Army Research Office Grant DAAG29-78-G-0042 at Research Triangle Park, N.C. 


\section{REFERENCES}

1. Bownds, J. M. Stability Implications on the Asymptotic Behavior of Second Order Differential Equations, Proc. Amer. Math. Soc. 39 (1973), 169-172, YR $47 \# 2150$.

2. Coppel, W. A. Stability and Asymptotic Behavior of Differential Equations, Heath, Boston, 1965. MR 32 \# 7875 .

3. Hatvani, L. and L. Pintér. On Pertubation of Unstable Second Order Linear Differential Equations, Proc. Amer. Math. Soc. 61 (1976), 36-38. 


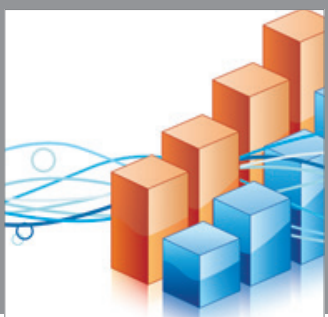

Advances in

Operations Research

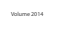

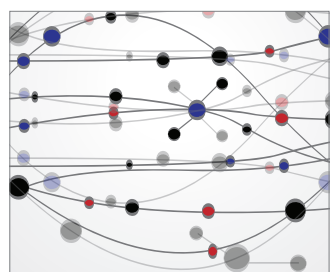

\section{The Scientific} World Journal
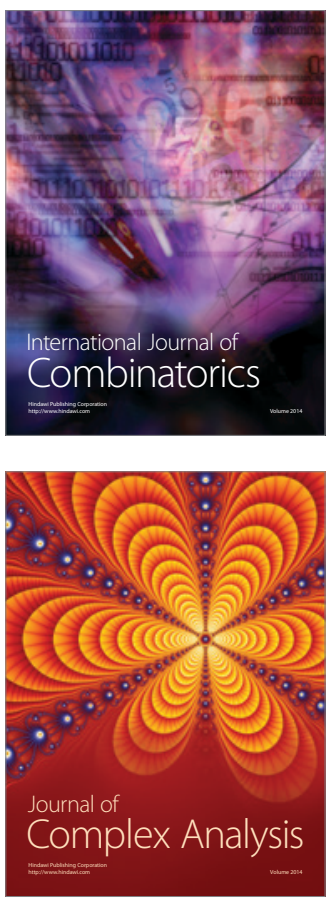

International Journal of

Mathematics and

Mathematical

Sciences
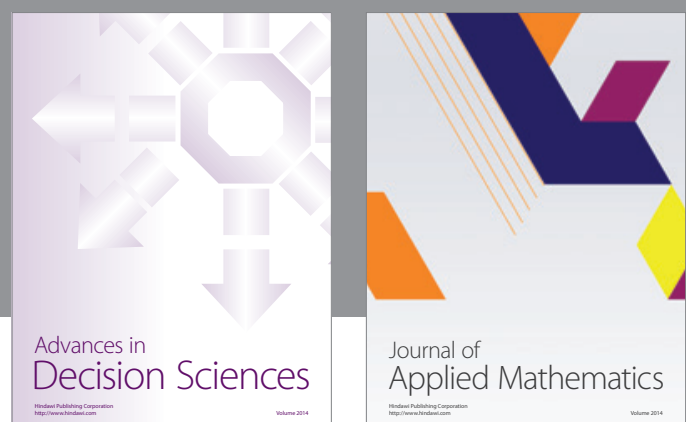

Journal of

Applied Mathematics
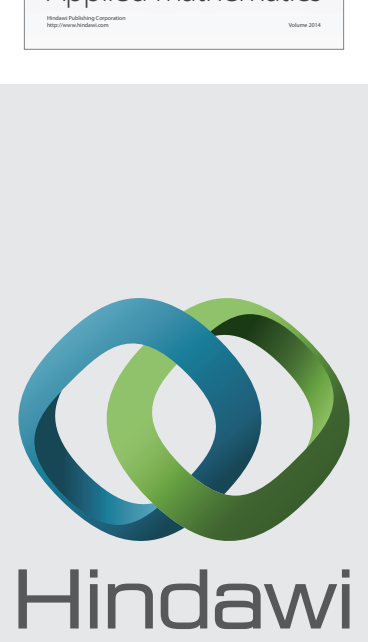

Submit your manuscripts at http://www.hindawi.com
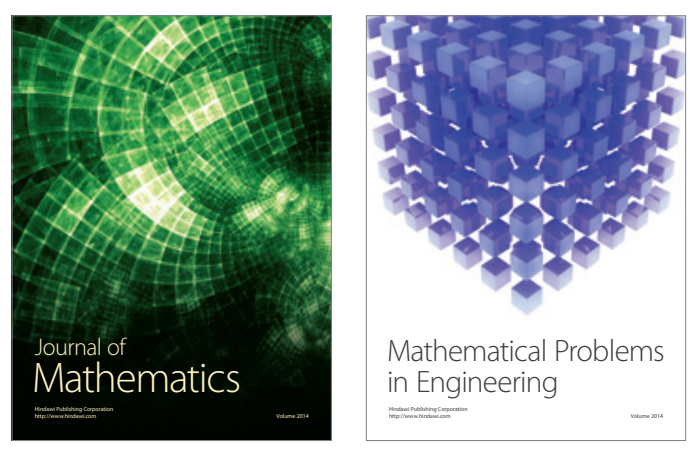

Mathematical Problems in Engineering
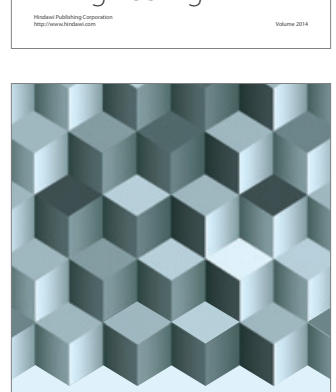

Journal of

Function Spaces
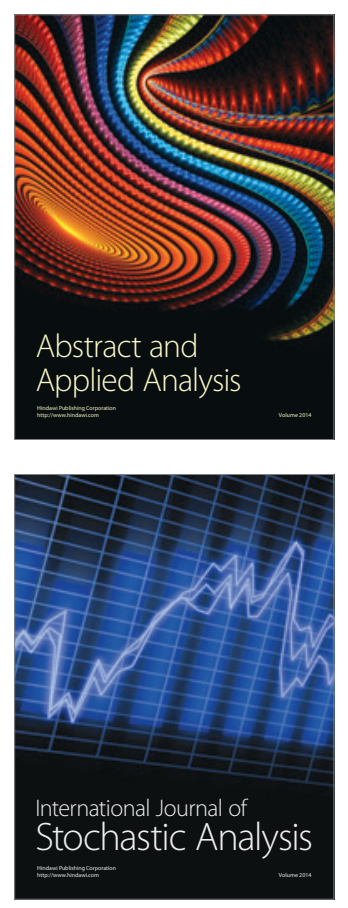

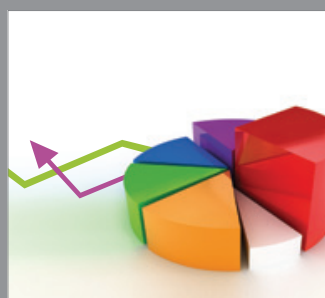

ournal of

Probability and Statistics

Promensencen
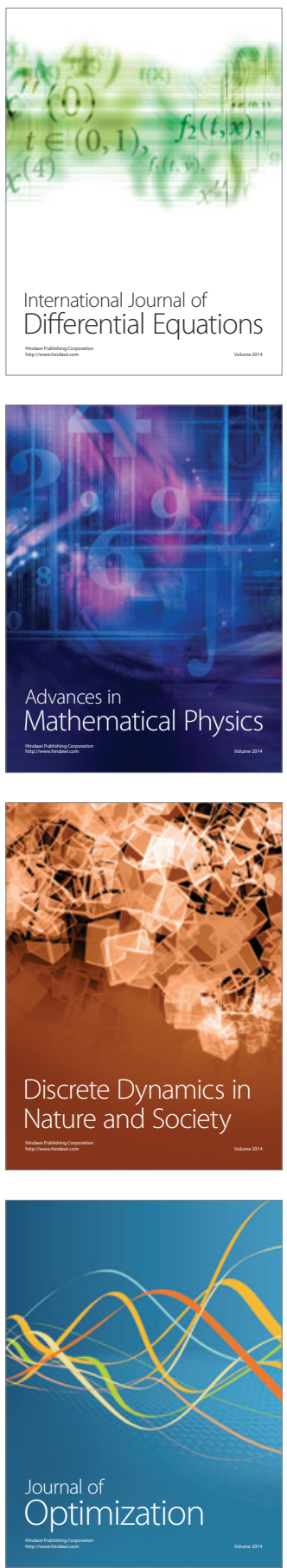